\title{
Performance Asymmetry in the Star Excursion Balance Test
}

Phillips, A., Condon, T., Keith, T., McKeon, P., King, D., Ithaca College, Ithaca, NY

The Star Excursion Balance Test (SEBT) is a common assessment used by clinicians to evaluate dynamic postural control. The SEBT incorporates aspects of strength, flexibility, and neuromuscular control. It often used to predict individuals at risk of certain lower extremity injury and to track rehabilitation progress. However, to better evaluate performance in clinical populations or identify injury risk, it is necessary to understand bi-lateral asymmetry in the SEBT in a healthy population. PUPROSE: To determine bilateral asymmetry in SEBT reach distances in healthy college aged individuals.

METHODS: Twenty-eight healthy male and female college aged students (Age: $19.8 \pm$ 1.0 yrs, Ht: $171.4 \pm 12.3 \mathrm{~cm}$, Mass: $78.7 \pm 22.6 \mathrm{~kg}$ ) performed 3 practice and 3 successful trials for the three directions of the SEBT: anterior (ANT), posterior-medial (PM), and posterior-lateral (PL) on their dominate (D) and non-dominate (ND) legs. Successful trials were defined as trials for which the subject, while performing their maximal reach, kept hands on hips, the balancing foot flat, and maintained static balance for 3 seconds after returning to the start position. The dominate leg was defined as their preferred kicking leg. Reach distance was recorded to the nearest $\mathrm{cm}$. A 3 trial mean was calculated for each subject, each distance, and each leg and used in all subsequent analyses. Bilateral asymmetries were evaluated using Bland-Altman Limits of Agreement analyses with $95 \%$ confidence intervals around the mean asymmetry. RESULTS: The anterior reach demonstrated the lowest mean asymmetry of the three reach distances but the greatest asymmetry variability $(0.9 \pm 4.3 \mathrm{~cm})$ with a potential 7.6 to $-9.4 \mathrm{~cm}$ asymmetry based on the $95 \%$ confidence intervals. The PM and PL demonstrated larger asymmetries (PM: $4.3 \pm 2.9 \mathrm{~cm}, \mathrm{P}: 3.6 \pm 2.2 \mathrm{~cm})$ with 1.3 to $-10.0 \mathrm{~cm}$ and 7.9 to $-0.8 \mathrm{~cm}$ asymmetries, respectively. Based on the LoA analysis, the PM asymmetry appeared to favor the dominant limb while the PL favored the non-dominant. CONCLUSION: Healthy college aged adults exhibited bi-lateral asymmetries during the SEBT when performing posterior reaches. These asymmetries appear to be limb dependent. Understanding asymmetries in healthy populations could lead to improved utilization of SEBT in clinical assessment. 